\title{
ON THE AVERAGE NUMBER OF OCTAHEDRAL MODULAR FORMS
}

\author{
Jordan S. ElLENBERG
}

Let $N>0$ be an integer, $\chi$ a Dirichlet character modulo $N$, and $k$ either 0 or 1 . Let $f$ be a primitive eigenform, not necessarily holomorphic, of level $N$ and Nebentypus $\chi$, and let $\lambda_{f}(n)$ be the eigenvalue of $T_{p}$ on $f$. We say $f$ is associated to a Galois representation $\rho: \operatorname{Gal}(\overline{\mathbb{Q}} / \mathbb{Q}) \rightarrow G L_{2}(\mathbb{C})$ if

$$
\begin{aligned}
\lambda_{f}(p) & =\operatorname{Tr}\left(\rho\left(\text { Frob }_{p}\right)\right) \\
\chi(p) & =\operatorname{det}\left(\rho\left(\text { Frob }_{p}\right)\right)
\end{aligned}
$$

for all $p$ not dividing $N$. Following [4], we define $S_{1 / 4, k}^{\operatorname{Artin}}(N, \chi)$ to be the finite set of primitive weight $k$ cupsidal eigenforms which admit an associated Galois representation. If $\rho: \operatorname{Gal}(\overline{\mathbb{Q}} / \mathbb{Q}) \rightarrow G L_{2}(\mathbb{C})$ is a Galois representation, we define $\mathbb{P} \rho$ to be the composition of $\rho$ with the natural projection $G L_{2}(\mathbb{C}) \rightarrow P G L_{2}(\mathbb{C})$.

Two-dimensional complex Galois representations fall naturally into four types; we call $\rho$ dihedral, tetrahedral, octahedral, or icosahedral according as the projectivized image $\mathbb{P} \rho(\operatorname{Gal}(\overline{\mathbb{Q}} / \mathbb{Q}))$ is isomorphic to a dihedral group, $A_{4}, S_{4}$, or $A_{5}$. Cusp forms associated to Galois representations are classified likewise.

The latter three types are called "exotic"; it is widely believed that the number of exotic cusp forms of level $N$ is at most $N^{\epsilon}$. The first results in this direction are due to Duke [3]. These results were later sharpened by Wong [6] and Michel and Venkatesh [4]. The latter authors proved that

$$
n^{\operatorname{tetr}}(N, \chi, k) \ll_{\epsilon} N^{2 / 3+\epsilon}, n^{\text {oct }}(N, \chi, k) \ll_{\epsilon} N^{4 / 5+\epsilon}, n^{\text {icos }}(N, \chi, k) \ll_{\epsilon} N^{6 / 7+\epsilon}
$$

where $n^{T}(N, \chi, k)$ is the number of weight $k$ cusp forms of level $N$, Dirichlet character $\chi$, and type $T$. (Note that $n^{T}(N, \chi, k)=0$ unless $\chi$ sends complex conjugation to $(-1)^{k}$.)

The goal of this paper is to show that the Michel-Venkatesh bound on octahedral forms can be sharpened on average over square-free levels $N$.

We begin by showing that, in the case of square-free level, one does not need to consider very many different Dirichlet characters $\chi$ when counting exotic cusp forms.

Lemma 1. Let $N$ range over square-free integers. Then the number of Dirichlet characters $\chi$ of conductor $N$ such that there exists an exotic cusp form of level $N$ and Nebentypus $\chi$ is $O\left(N^{\epsilon}\right)$.

Received December 31, 2002.

Partially supported by NSA Young Investigator Grant MDA905-02-1-0097. 
Proof. Let $\rho$ be the Galois representation attached to an exotic cusp form of level $N$ and conductor $\chi$. Let $p$ be a prime dividing $N$. Then, since $p \| N$, the restriction $\rho: I_{p} \rightarrow G L_{2}(\mathbb{C})$ must decompose as $\chi \oplus 1$. In particular, the projection from $\rho\left(I_{p}\right)$ to $\mathbb{P} \rho\left(I_{p}\right)$ is an isomorphism. So $\chi\left(I_{p}\right)$ is a cyclic subgroup of either $A_{4}, S_{4}$, or $S_{5}$; in particular, $\chi^{60}$ is unramified everywhere, whence trivial. Now the number of characters $\chi$ of level $N$ such that $\chi^{60}=1$ is $O\left(N^{\epsilon}\right)$, which proves the lemma.

Suppose from now on that $N$ is square-free. Let $n^{\text {oct }}(N)$ be the number of octahedral cusp forms of level $N$. It follows from Lemma 1 and the theorem of Michel and Venkatesh that

$$
n^{\text {oct }}(N) \ll_{\epsilon} N^{4 / 5+\epsilon} .
$$

Let $p: S_{4} \rightarrow S_{3}$ be the natural surjection. Then every homomorphism $\operatorname{Gal}(\overline{\mathbb{Q}} / \mathbb{Q}) \rightarrow S_{4}$ can be composed with $p$ to yield a homomorphism $\psi$ : $\operatorname{Gal}(\overline{\mathbb{Q}} / \mathbb{Q}) \rightarrow S_{3}$. By combining arguments from [6] and [4], we obtain the following sharpening of Theorem 10 of [6]:

Proposition 2. Let $k$ be 0 or 1 , and $N$ a positive square-free integer. Let $\psi: \operatorname{Gal}(\overline{\mathbb{Q}} / \mathbb{Q}) \rightarrow S_{3}$ be a homomorphism, and let $n_{\psi}^{\text {oct }}(N)$ be the number of octahedral weight $k$ cusp forms associated to Galois representations $\rho$ such that $p \circ \mathbb{P} \rho=\psi$.

Then $n_{\psi}^{o c t}(N) \ll_{\epsilon} N^{2 / 3+\epsilon}$.

Proof. Let $\chi$ be a character of level $N$. In the proof of [6, Thm. 10], Wong constructs an amplifier-that is, a set of complex numbers $\left\{c_{n}\right\}_{n \in \mathbb{N}}$ such that, for some absolute constants $C$ and $C^{\prime}$,

- $\sum_{n \leq B}\left|c_{n}\right| \leq C B^{1 / 4}$.

- $\sum_{n \leq B}\left|c_{n}\right|^{2} \leq C B^{1 / 4}$.

- If $\rho$ is an octahedral Galois representation such that $p \circ \mathbb{P} \rho=\psi$, and $f$ is a cusp form in $S_{1 / 4, k}^{\operatorname{Artin}}(N, \chi)$ associated to $\rho$, then $\left|\sum_{n \leq B} c_{n} \lambda_{f}(n)\right| \geq$ $C^{\prime} B^{1 / 4} / \log B$.

In $[4, \S 3]$, Michel and Venkatesh use the Petersson-Kuznetzov formula and standard bounds on Kloosterman sums to obtain the following inequality:

$$
\begin{aligned}
\sum_{f \in \Sigma}(f, f)^{-1}\left|\sum_{\substack{n \leq B \\
(n, N)=1}} c_{n} \lambda_{f}(n)\right|^{2} & \ll_{\epsilon} \sum_{\substack{n \leq B \\
(n, N)=1}}\left|c_{n}\right|^{2}+(B N)^{\epsilon} B^{1 / 2} N^{-1}\left(\sum_{\substack{n \leq B \\
(n, N)=1}}\left|c_{n}\right|\right)^{2}
\end{aligned}
$$


where

- $\Sigma$ is a set of eigenforms in $S_{1 / 4, k}^{\operatorname{Artin}}(N, \chi)$;

- $(f, f)$ is the Petersson self-product of $f$;

- $\left\{c_{n}\right\}$ is an arbitrary sequence of complex numbers.

Speaking loosely, the idea of [4] and [3] is that, by the Petersson-Kuznetzov formula, the vectors $\left\{\lambda_{f}(n)\right\}_{f \in \Sigma}$ and $\left\{\lambda_{f}(m)\right\}_{f \in \Sigma}$ are "approximately orthogonal" when $m$ and $n$ are distinct integers. On the other hand, Wong shows that there exists a large set of $n$ such that the Fourier coefficients $\lambda_{f}(n)$ are real numbers of a fixed sign as $f$ ranges over $\Sigma$. The desired bound on $\Sigma$ will follow from the tension between these two constraints.

In the above inequality, take $\left\{c_{n}\right\}$ to be Wong's amplifier and $\Sigma$ to be the set of octahedral forms in $S_{1 / 4, k}^{\operatorname{Artin}}(N, \chi)$ associated to Galois representations $\rho$ such that $p \circ \mathbb{P} \rho=\psi$.

Note that $(f, f)=O\left(N \log ^{3} N\right)$ by $[6$, Lemma 6$]$. So the left hand side of $(1)$ is bounded below by a constant multiple of

$$
N^{-1} \log ^{-3} N \sum_{f \in \Sigma}\left|\sum_{\substack{n \leq B \\(n, N)=1}} c_{n} \lambda_{f}(n)\right|^{2} \geq N^{-1} \log ^{-3} N|\Sigma|\left(C^{\prime}\right)^{2} B^{1 / 2} \log ^{-2}(B)
$$

while the right hand side is bounded above by a constant multiple of

$$
B^{1 / 4}+(B N)^{\epsilon} B^{1 / 2} N^{-1} B^{1 / 2} .
$$

Combining these bounds, one has

$$
|\Sigma| \ll_{\epsilon} N \log ^{3}(N) B^{-1 / 2} \log ^{2}(B)\left(B^{1 / 4}+(B N)^{\epsilon} B N^{-1}\right)
$$

The bound is optimized when we take $B \sim N^{4 / 3}$, which yields

$$
|\Sigma| \ll_{\epsilon} N^{2 / 3+\epsilon} \text {. }
$$

Combined with the fact that the number of $\chi$ under consideration is $O\left(N^{\epsilon}\right)$, this yields the desired result.

Proposition 2, in combination with the theorem of Davenport and Heilbronn on cubic fields, allows us to improve Michel and Venkatesh's bound on $n^{\text {oct }}(N)$ in the average.

Theorem 3. For all $\epsilon>0$ there exists a constant $C_{\epsilon}$ such that

$$
(1 / X) \sum_{\substack{N<X \\ N \text { sq.free }}} n^{\text {oct }}(N)<C_{\epsilon} X^{2 / 3+\epsilon}
$$

for all $X>1$.

Proof. Let $f$ be an octahedral form of level $N$ associated to a representation $\rho$. For each prime $p \mid N$, the group $\mathbb{P} \rho\left(I_{p}\right)$ is a cyclic subgroup of $S_{4}$. (Recall that $N$ is square-free.) Define 
- $N_{1}$ to be the product of primes $p$ such that $\mathbb{P} \rho\left(I_{p}\right)$ is a nontrivial subgroup of the Klein four-group;

- $N_{2}$ to be the product of primes $p$ such that $\mathbb{P} \rho\left(I_{p}\right)$ is contained in $A_{4}$ but not in the Klein four-group;

- $N_{3}$ to be the product of primes $p$ such that $\mathbb{P} \rho\left(I_{p}\right)$ is not contained in $A_{4}$.

Then $N_{1} N_{2} N_{3}=N$. Let $\psi: \operatorname{Gal}(\overline{\mathbb{Q}} / \mathbb{Q}) \rightarrow S_{3}$ be the composition $p \circ \mathbb{P} \rho$. Then the fixed field $L$ of $\operatorname{ker} \psi$ is a cyclic 3-cover of a quadratic number field $K$, where $K / \mathbb{Q}$ is the unique quadratic field ramified precisely at primes dividing $N_{3}$, and $L / K$ is ramified only at primes dividing $N_{2}$.

Let $b\left(N_{2}, N_{3}\right)$ be the number of such $S_{3}$-extensions $L$. (For notational convenience we take $b\left(N_{2}, N_{3}\right)$ to be 0 when either $N_{2}$ or $N_{3}$ is not square-free.) From Proposition 2, we have

$$
n^{\text {oct }}(N) \ll_{\epsilon} \sum_{\substack{N_{1}, N_{2}, N_{3} \\ N_{1} N_{2} N_{3}=N}} b\left(N_{2}, N_{3}\right) N^{2 / 3+\epsilon} .
$$

Let $T$ be the set of places of $K$ dividing $3 N_{2} \infty$, and let $G_{T}(K)$ be the Galois group of the maximal extension of $K$ unramified away from $T$. Each cubic field counted in $b\left(N_{2}, N_{3}\right)$ is a cyclic 3 -extension of $K$ unramified away from $T$, so

$$
b\left(N_{2}, N_{3}\right) \leq\left|\operatorname{Hom}\left(G_{T}(K), \mathbb{Z} / 3 \mathbb{Z}\right)\right| .
$$

The Galois cohomology group above fits in an exact sequence

$$
\begin{aligned}
0 \rightarrow \operatorname{Hom}\left(\mathrm{Cl}_{T}(K), \mathbb{Z} / 3 \mathbb{Z}\right) \rightarrow \operatorname{Hom}\left(G_{T}(K), \mathbb{Z} / 3 \mathbb{Z}\right) & \\
& \rightarrow \prod_{v \in T} \operatorname{Hom}\left(\operatorname{Gal}\left(\bar{K}_{v} / K_{v}\right), \mathbb{Z} / 3 \mathbb{Z}\right)
\end{aligned}
$$

where $\mathrm{Cl}_{T}(K)$ is the quotient of the class group of $K$ by all primes in $T$. (See [5, (8.6.3)]). Let $h_{3}\left(N_{3}\right)$ be the order of the 3 -torsion subgroup of the class group of $K$. Since $\operatorname{dim}_{\mathbb{F}_{3}} \operatorname{Hom}\left(\operatorname{Gal}\left(\bar{K}_{v} / K_{v}\right), \mathbb{Z} / 3 \mathbb{Z}\right)$ is at most 4 (see $[5,(7.3 .9)]$ ), we have

$$
b\left(N_{2}, N_{3}\right) \leq h_{3}\left(N_{3}\right) 3^{4|T|} \ll_{\epsilon} N_{2}^{\epsilon} h_{3}\left(N_{3}\right) .
$$

Combining (3) and (2) yields

$$
n^{\text {oct }}(N) \ll_{\epsilon} \sum_{\substack{N_{1}, N_{2}, N_{3} \\ N_{1} N_{2} N_{3}=N}} h_{3}\left(N_{3}\right) N^{2 / 3+\epsilon} .
$$

Since the sums over $N_{1}$ and $N_{2}$ have length at most $d(N)=O\left(N^{\epsilon}\right)$, we have

$$
n^{\text {oct }}(N) \ll_{\epsilon} \sum_{N_{3} \mid N} h_{3}\left(N_{3}\right) N^{2 / 3+\epsilon} .
$$


So

$$
\begin{aligned}
\sum_{\substack{N<X \\
N \text { sq.free }}} n^{\text {oct }}(N) \ll_{\epsilon} \sum_{N_{3}=0}^{X} h_{3}\left(N_{3}\right) \sum_{k=0}^{X / N_{3}}\left(k N_{3}\right)^{2 / 3+\epsilon} & \\
& \leq X^{5 / 3+\epsilon} \sum_{N_{3}=0}^{X} h_{3}\left(N_{3}\right)\left(1 / N_{3}\right) .
\end{aligned}
$$

The sum $\sum_{d=0}^{X} h_{3}(d) / d$ can be estimated as follows. Integration by parts yields

$$
\sum_{d=0}^{X} h_{3}(d) / d=(1 / X) \sum_{d=0}^{X} h_{3}(d)+\int_{1}^{X}\left(\sum_{d=1}^{t} h_{3}(d)\right) t^{-2} d t
$$

Now by the the theorem of Davenport and Heilbronn [2, Theorem 3] we have $\sum_{d=0}^{t} h_{3}(d)=O(t)$. It follows that

$$
\sum_{d=0}^{X} h_{3}(d) / d=O(\log X)
$$

Substituting this bound into (4) gives

$$
\sum_{\substack{N<X \\ N \text { sq.free }}} n^{\text {oct }}(N) \ll_{\epsilon} X^{5 / 3+\epsilon}
$$

which yields the desired result.

Theorem 3 can be thought of as a bound for the number of quartic extensions of $\mathbb{Q}$ whose Artin conductor, with respect to a certain 2-dimensional projective representation of $S_{4}$, is bounded by $X$. This is quite different from the problem, recently solved by Bhargava [1], of counting the number of quartic extensions of $\mathbb{Q}$ with discriminant less than $X$. For instance, quartic extensions attached to cusp forms of conductor $N$ might have discriminant as large as $N^{3}$.

\section{References}

[1] M. Bhargava, Higher composition laws, PhD thesis, Princeton, 2001.

[2] H. Davenport, H. Heilbronn, On the density of discriminants of cubic fields. II, Proc. Roy. Soc. London Ser. A 322 (1971), 405-420.

[3] W. Duke, The dimension of the space of cusp forms of weight one, Internat. Math. Res. Notices 1995, 99-109.

[4] P. Michel, A. Venkatesh, On the dimension of the space of cusp forms associated to 2dimensional complex Galois representations, Int. Math. Res. Not. 2002, 2021-2027.

[5] J. Neukirch, A. Schmidt, K. Wingberg, Cohomology of number fields, Grundlehren der Mathematischen Wissenschaften, 323. Springer-Verlag, Berlin, 2000.

[6] S. Wong, Automorphic forms on $\mathrm{GL}(2)$ and the rank of class groups, J. Reine Angew. Math. 515 (1999), 125-153.

Princeton University Mathematics Department, Fine Hall, Washington Road, PRINCETON NJ 08544-1000, U.S.A.

E-mail address: ellenber@math.princeton.edu 